\title{
Radioimmunotherapy - A New Approach in the Management of Non-Hodgkin Lymphoma
}

a report by

Timothy M IIIidge

Professor, Targeted Therapy and Oncology, Christie Hospital, Manchester

DOI: $10.17925 / E O H \cdot 2005 \cdot 0.0 .85$

Despite the sensitivity of most lymphomas to initial therapy with chemotherapy or radiotherapy, the majority of patients with advanced non-Hodgkin's lymphoma (NHL) eventually relapse and die of their disease. ${ }^{1}$ Furthermore, patients with advanced low-grade lymphomas remain incurable and their survival has not altered since the early 1960s. ${ }^{2}$ The introduction of antibody (mAb)-based therapy initially with Rituximab in the late 1990s and, more recently, with the conjugation of radioisotopes to $\mathrm{mAb}$ as part of radioimmunotherapy (RIT) has provided fresh hope for NHL patients that their prognosis can be improved. Rituximab has now become integrated in the treatment of most NHL and while the response rates of rituximab as a single agent remain modest, with complete response rates in single figures, ${ }^{4}$ combining rituximab with combination chemotherapy (CHOP) has shown a survival advantage when used in the treatment of aggressive lymphomas, and dramatic improvements in progression-free survival. ${ }^{5-7}$

To date, antibodies directed towards the CD20 antigen have dominated the field of $\mathrm{mAb}$ therapy and RIT of lymphoma. CD20 is highly expressed on mature B-cells and present on 95\% of B-cell lymphomas. ${ }^{8,9}$ It is neither internalised nor shed from the cell surface and appears on binding $\mathrm{mAb}$ to initiate signalling and trigger apoptosis through a caspase-dependent pathway. ${ }^{9}$

${ }^{131}$ I labelled Tositumomab (Bexxar ${ }^{\mathrm{TM}}$ ) and ${ }^{90} \mathrm{Y}$ labelled ibritumomab tiuxetan $\left(\right.$ Zevalin $\left.^{\circledR}\right)$ are highly promising therapies with significantly increased overall and complete response rates over Rituximab. Both drugs appear able to offer long durable remissions for some patients. As Zevalin is the first and only one of this class of radioimmunconjugates to have been granted EU approval (May 2004), this brief article will be limited to describing Zevalin. The introduction of Zevalin has some parallels with the initial introduction of Rituximab into clinical practice in the late 1990s. There is no doubting that this is an active drug but considerable uncertainty remains as to when and how to best integrate Zevalin into clinical practice even within the licensed indication of relapsed follicular lymphoma. Furthermore, there are many new opportunities to explore integrating Zevalin into the treatment of other NHL. Over the last year, encouraging data has emerged that suggests that Zevalin may play a useful role as consolidation after brief chemotherapy for untreated follicular lymphoma. It can form a component in the treatment of diffuse large B-cell lymphoma (DLBCL) and may be integrated into the conditioning regimen for autologous stem cell

1. Armitage J O, "Treatment of Non-Hodgkin's Lymphoma", N Engl J Med (1993);328: pp. 1,023-1,030.

2. Horning $S J$, "Natural history of and therapy for the indolent non-Hodgkin's lymphomas", Semin Oncol (1993);20: pp. $75-88$.

3. Kohler G, Milstein C, "Continuous cultures of fused cells secreting antibody of predefined specificity", Nature (1975);256: pp. 495-497.

4. McLaughlin P et al., "Rituximab chimeric anti-CD20 monoclonal antibody therapy for relapsed indolent lymphoma", J Clin Oncol (1998);16: pp. 2,825-2,833.

5. Coiffier $B$ et al., "CHOP chemotherapy plus rituximab compared with $C H O P$ alone in elderly patients with diffuse large b-cell lymphoma”, N Engl J Med (2002);346: pp. 235-224.

6. Coiffier B, "Rituximab in combination with CHOP improves survival in elderly patients with aggressive non-Hodgkin's", Semin Oncol (2002);29: pp. 18-22.

7. Marcus et al., "CVP chemotherapy plus rituximab compared to CVP as first-line treatment for advanced follicular lymphoma”, Blood (2005);105(4): pp. 1,417-1,423.

8. Grossbard ML et al., "Monoclonal antibody-based therapies of leukemia and lymphoma", Blood (1992);80: pp. 863-878.

9. Press $O W$ et al., "Comparative metabolism and retention of iodine-125, yttrium-90, and indium-111 radioimmunoconjugates by cancer cells”, Cancer Res (1996);56: pp. 2,123-2,129.

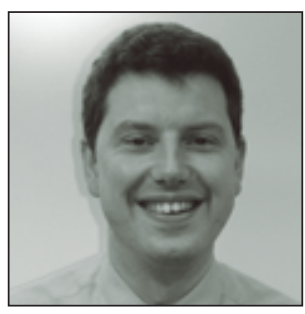

Timothy M Illidge, MD, PhD, FRCR, FRCP, is Professor of Targeted Therapy and Oncology and Cancer Research UK (CRUK) Senior Clinical Research Fellow at the CRUK Paterson Institute and Christie Hospital in Manchester, UK. He serves on the radiotherapy and lymphoma groups of the National Cancer Research Institute and is chairman of the research committee of the Royal College of Radiologists. He currently leads the pre-clinical and clinical radioimmunotherapy programme at the Christie Hospital at Manchester University, where he has a special interest in the management of lymphoma. Dr Illidge completed research fellowships at the Cancer Research Council (UK) where he was Senior Lecturer and Honorary Consultant in Oncology at Southampton University Hospitals NHS Trust, and at Stanford University, California, where he was a member of the clinical radioimmunotherapy programme. After completing an undergraduate degree at London University, he received his medical degree from Guy's Hospital Medical School (London) and his PhD from the University of Southampton (UK). 


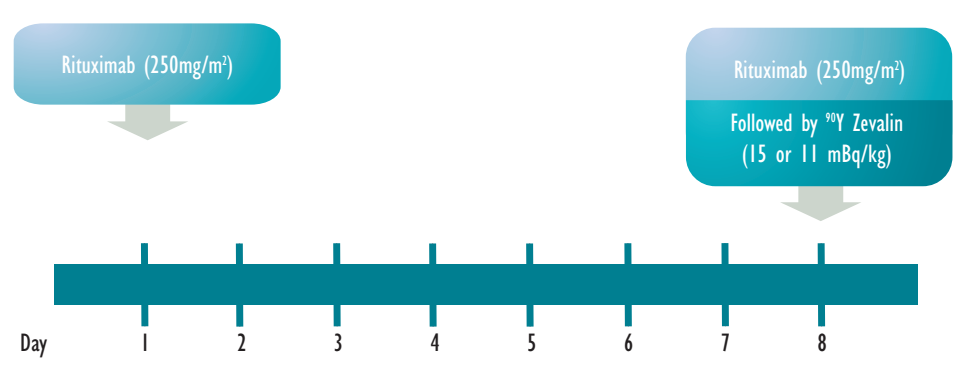

Table I: Results of Initial Studies of Zevalin

\begin{tabular}{llll} 
& Phase IIII & Phase II & Phase III \\
\hline & $\mathbf{n = 5 I}$ & $\mathbf{n = 3 0}$ & $\mathbf{n = 7 3}$ \\
\hline Overall Response \% & 73 & 83 & 80 \\
\hline Median DR (months) & $I I .7$ & $I I .5$ & 13.9 \\
\hline CR, Cru \% & $29 *$ & 47 & 34 \\
\hline Median DR (months) & $28^{*}$ & 23 & 23 \\
\hline On-going CR, Cru \% & 19 & 14 & 32 \\
\hline Median DR (months) & 62.1 & $4 I .2$ & 42.2 \\
\hline Range & $60+$ to $66+$ & $40+$ to $42+$ & $33+$ to $48+$ \\
\hline
\end{tabular}

Witzig et al., Proceedings of ASCO 2003.

Gordon et al., "Durable responses after ibritumomab tiuxetan radioimmunotherapy for CD20+ B-cell lymphoma: long-term follow-up of a phase I/2 study", Blood (2004); 103(15): pp. 4,429-4,43I.

Figure 2: Stages to Zevalin Treatment

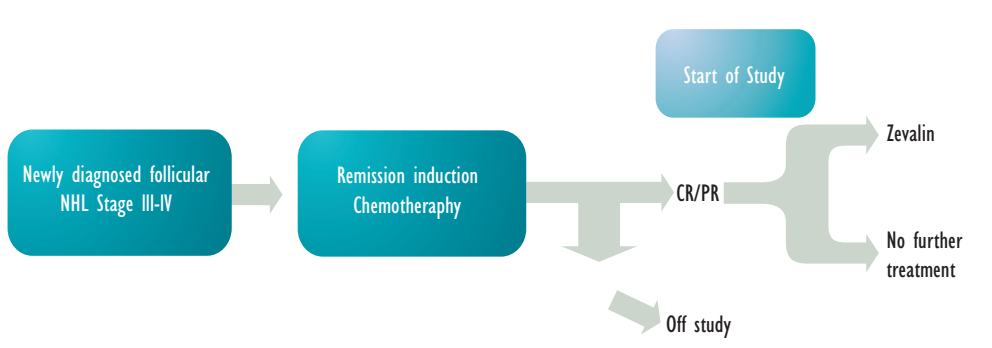

transplant instead of total body irradiation (TBI).

What is Zevalin RIT?

Zevalin is composed of the monoclonal antibody ibritumomab, covalently bound to tiuxetan, a highaffinity chelator for the radioisotope ${ }^{90} \mathrm{Y}$. Ibritumomab is a murine $\operatorname{IgG} 1$ kappa monoclonal antibody that specifically targets the CD20 antigen. ${ }^{10}$ The tiuxetan chelator creates a high-affinity, stable urea-type bond between the antibody and the radioisotope, to prevent the radioisotope dissociating and circulating around the body. ${ }^{11}$

The Zevalin treatment consists of a pre-treatment of the rituximab. The regimen is delivered over seven to nine days on an out-patient basis. A typical course of treatment involves a one-day intravenous (IV) infusion of rituximab $250 \mathrm{mg} / \mathrm{m}^{2}$; a second IV infusion of rituximab on day seven, eight or nine; immediately followed by an IV 'slow push' 10-minute infusion of ${ }^{90} \mathrm{Y}$ labelled Zevalin. By labelling mAbs with betaemitting radioisotopes, radiation can be targeted to the tumour increasing the potential for clinical response. Lymphomas are highly radiosensitive tumours and localized irradiation is the only treatment that potentially offers curative treatment for those patients with early-stage low-grade lymphomas. ${ }^{11}$ RIT has the potential advantage over other $\mathrm{mAb}$ directed therapy that it may kill tumour cells not directly targeted by the $\mathrm{mAb}$ via the 'crossfire effect'. This can provide cell kill to adjacent, antigen negative tumour cells. ${ }^{11}$

The unlabeled antibodies may bring about anti-tumor effects not only by recruiting the host immune system but also through inducing cell cycle arrest or apoptosis. Pre-clinical work indicates that intrinsic cytotoxicity of mAb in RIT may be as important as its ability to effectively deliver targeted radiotherapy. ${ }^{12,13}$ This suggests that there may be a synergistic interaction between the $\mathrm{mAb}$ effector mechanisms and the radiation.

${ }^{90}$ Yttrium (now licensed for use with Zevalin under the brand name Yttracis ${ }^{\circledR}$ ) offers a number of advantages over the most commonly used radioisotope in oncology practice, ${ }^{131}$ Iodine. ${ }^{90} \mathrm{Y}$ is a pure beta emitter delivering higher energy radiation $(2.3 \mathrm{MeV} \vee 0.6 \mathrm{MeV})$ at a longer path length $(5.3 \mathrm{~mm}$ versus $0.8 \mathrm{~mm})$. This enhances the crossfire effect and may be advantageous in treating larger, less well-vascularized tumour nodules. ${ }^{9}$ The physical half-life is 64 hours, which matches the biological half-life of murine monoclonal antibody of Zevalin, and the absence of penetrating gamma emissions enables delivery as an out-patient.

Zevalin is dosed according to the patient's body weight and baseline platelet counts. For patients with platelet counts $\geq 150,000 / \mathrm{mm}^{3}, 5 \mathrm{MBq} / \mathrm{kg}$ body weight is given, up to a maximum allowable dose of $1,200 \mathrm{MBq}$. For patients with platelet counts of 100,000$149,000 / \mathrm{mm}^{3}$, Zevalin is dosed at $11 \mathrm{MBq} / \mathrm{kg}$, up to a maximum allowable dose of $1,200 \mathrm{MBq}$.

\section{Clinical Experience with Zevalin RIT}

Zevalin RIT has been used for over 10 years and has emerged as a safe, effective and well tolerated therapy for relapsed 'low-grade' NHL. ${ }^{11}$ The majority of patients treated in the registration

10. Witzig et al., "Randomised controlled trial of ytrrium-90 labelled ibritumomab tiuxetan", J Clin Oncol 220 pp. 2, 453-2, 463 .

11. Gordon L I, et al., "Yttrium 90 ibritumomab tiuxetan radioimmunotherapy for relapsed or refractory low-grade nonHodgkin's lymphoma", Semin Oncol (2002); 29: pp. 87-92. 


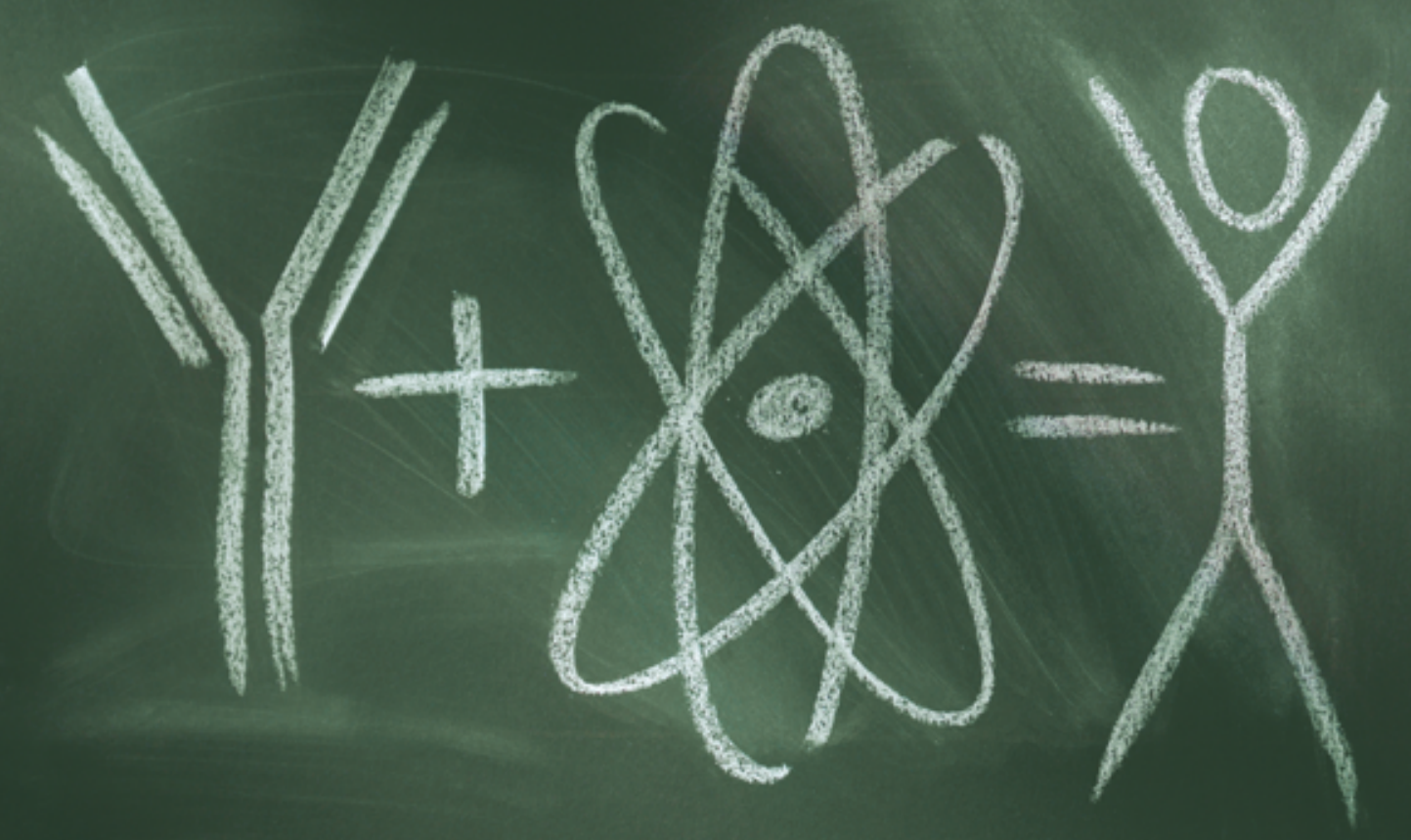

\section{United for success against NHL}

- The first radioimmunotherapy for follicular NHL

- High efficacy with more durable remissions and response rates significantly superior to antibody treatment alone

Convenient treatment and generally well tolerated

Name of the medicinal product Zevalin ${ }^{\circledast} 1.6 \mathrm{mg} / \mathrm{ml}$, kit for radiopharmaceutical preparation for infusion. Qualitative and $2 \mathrm{mg}$ of ibritumomab tiuxetan. Zevalin ${ }^{\circledR}$ is supplied as a kit for the preparation of yttrium-90 radiolabelled ibritumomab tiuxetan. The final formulation atter radiolabelling contains $2.08 \mathrm{mg}$ ibritumomab tiuxetan in a total volume of $10 \mathrm{ml}$. "produced by a genetically engineered Chinese Hamster Ovary (CHO) cell line conjugated to the chelating agent MX-DTPA Therapeutic indication The 90 -radiolabelled Zevalin ${ }^{\otimes}$ is indicated for the treatment of adult patients with rituximab relapsed or refractory CD20+ follicular B-cell non-Hodgkin's lymphoma (NHL). Contraindications Hypersensitivity to ibritumomab tuxetan, to ytrium chloride, to other murine proteins or to any of the excipients. Pregnancy and lactation. Undesirable effects The frequencies of the adverse reactions reported below (very common $\geq 10 \%$, common $\geq 1$ to $<10 \%$, uncommon $<1 \%$ ) are based on clinical trial data irrespective of causality. Anaphylactic reactions and hypersensitivity. Anaphylactic and other hypersensitivity reactions have been reported in less than $1 \%$ of patients following the intravenous administration of proteins to patients. Medicinal products for the treatment of hypersensitivity reactions, e.g. adrenaline, antihistamines and corticosteroids, should be avaliable for immediate use in the event of an allergic reaction during administration of Zevalin ${ }^{\circledR}$. Haematological adverse reactions. Haematological toxicity has been very commonly observed in clinical trials, and is dose-limiting Median time to blood platelet and granulocyte nadirs wore around 60 days after start of treatment Grade 3 or 4 thrombocytopenia was reported with median times to

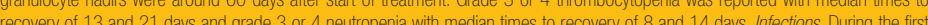

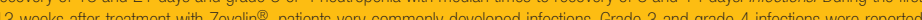
13 weeks atter treatment with Zevalin, patients very commonly developed infections. Grade 3 and grade 4 infections were reported Myelodysplasia/acute myeloid leukaemia (AML) has been reported in five out of 211 patients assigned to treatment with Zevalin ${ }^{\circledR}$. The risk of developing secondary myelodysplasia or leukaemia following therapy with alkylating agents is well known. Since all of these patients were pre-treated with alkylating agents, avaliable results provide insufficient data on whether Zevalin ${ }^{\oplus}$ contributes to an increased risk of myelodysplasia, or on the extent of risk. Adverse reactions by body system. Body as a whole, very common: asthenia, infection, chills, fever, abdominal pain, headache, pain, throat irritation, back pain; common: flushing, allergic reactions, chest pain, neck pain, malaise, tumour pain, abdominal enlargement, flu syndrome, moniliasis (candidiasis), mucous membrane disorder, axilla pain, pelvis pain, sepsis. Cardiovascular system, common: hypotension, hypertension, tachycardia, pallor, palpitation. Digestive system, very common: nausea, vomiting, diarhoea, anorexia; common: constipation, dyspepsia, dry mouth, gastrointestinal disorder, stomatitis, rectal haemormage, gum haemorrhage, melaena. Injection site, common: pain at injection site. Metabolic and nutritional disorders, common: peripheral oedema, angio-oedema, increased LDH, dehydration, hyperglycaemia, increased alkaline phosphatase, oedema, increased SGOT. decreased weight, increased blood urea nitrogen, hypocalcaemia, increased SGPT. Musculoskeletal system, very common: arthralgia: common: myalgia, bone pain, leg cramps, myasthenia. Haemic and lymphatic system, very common: thrombocytopenia, anaemia, leukocytopenia, granulocytopenia, ecchymosis; common: petechia, hypochromic anaemia, febrile neutropenia. Nervous system, very common: dizziness: common: insomnia, anxiety depression, paresthesia, hypesthesia somnolence vasodilatation, abnormal gait Respiratory system, very common: dyspnoea, increased cough; common: minitis, infection, bronchospasm, sinusitis, epistaxis, bronchitis, voice alteration, chest pain, pharyngitis, pneumonia. Skin and appendages, very common: pruritus; common: rash, urticaria, sweating. sweating at night, skin disorder, herpex simplex. Special senses, common: conjunctivits, amblyopia, abnormal vision. Urogenital system, common: urinary tract infection, dysuria. Date of revision of the text January 2004 Please note! For current prescribing common: urinary tract infection, dysuria. Date of revision of the text January 2004 Please note! For current presc
information refer to the package insert and/or contact your local Schering organisation. Schering AG, 13342 Berlin, Germany 
approval studies had relapsed follicular lymphoma. These published studies consistently show high rates of overall and complete response rates in relapsed follicular lymphoma.

Even in previously heavily pre-treated patients who had received a median of four different chemotherapy regimens and who either did not respond to prior rituximab therapy or had disease progression within six months of therapy, the overall response rate of $74 \%$, with $15 \%$ complete response was seen. ${ }^{11} \mathrm{~A}$ randomized controlled trial of Zevalin versus rituximab in relapsed or refractory low-grade or transformed follicular B-cell NHL was performed with the primary aim of demonstrating superior response rates of Zevalin over Rituximab. ${ }^{10}$ Seventythree patients received two doses of rituximab $250 \mathrm{mg} / \mathrm{m}^{2}$ a week apart as pre-dosing followed by a single dose of Zevalin $0.4 \mathrm{mCi} / \mathrm{kg}(15 \mathrm{Mbq} / \mathrm{kg})$. Seventy patients in the control arm received rituximab $375 \mathrm{mg} / \mathrm{m}^{2}$ weekly for four weeks. The overall response rate was $80 \%$ for the Zevalin group versus $56 \%$ for the rituximab alone group $(p=0.002)$. Complete responses were $30 \%$ and $16 \%$ in the Zevalin and rituximab groups, respectively. Both regimens were well tolerated but, as expected, there was more myelosuppression in the RIT group. had become refractory to chemotherapy, which was significantly greater than that seen with Rituximab. This data suggests the Zevalin offers hope for meaningful clinical responses even in patients who have become refractory to chemotherapy.

Perhaps the most impressive finding to emerge from these initial studies was that around $70 \%$ of the patients who achieve a CR remain in remission for years - some patients treated in the early studies are now in remission for more than five years after Zevalin treatment, with a median follow-up of almost four years (see Table 1). ${ }^{11}$

An analysis of prognostic factors has confirmed that this remarkable durability of response is unlikely to be accounted for by patient selection as most of these durable remissions have been achieved in heavily chemotherapy pre-treated and chemorefractory patients with validated poor prognostic factors, such as extensive prior therapy (one to nine regimens), bulky disease, high lactate dehydrogenase (LDH) and extranodal disease. Only disease bulk correlated with the overall response rate $(<5 \mathrm{~cm}) \quad(89$ patients objective response rate (ORR) 90\% $(\mathrm{p}<0.001) .{ }^{12}$

\section{Zevalin is composed of the monoclonal antibody ibritumomab, covalently bound to tiuxetan, a high-affinity chelator for the radioisotope ${ }^{90} Y$.}

These highly promising results demonstrated that Zevalin led to superior overall and complete response rates to those seen with Rituximab, and for patients with follicular lymphoma this translated into a significant improvement in the time to treatment failure. An interesting and potentially important finding to emerge from this study was the high level of activity of Zevalin in patients who
Examining the toxicity seen for patients treated in the Zevalin trials $(n=261)$ indicates that $28 \%$ will experience grade 4 neutropenia and $8 \%$ will experience grade 4 thrombocytopenia. ${ }^{11}$ The nonhematological toxicity is extremely modest and even the hematological toxicity can be minimized if appropriate precautions are taken. Patients who have been heavily treated with chemotherapy, such

12. Du Y, Honeychurch J, Bayne M, et al., "Antibody induced intracellular signaling works in combination with radiation to eradicate lymphoma in radioimmunotherapy", Blood (2004);103: pp. 1,485-1,494.

13. Illidge T M, Cragg MS, McBride H, et al., "The importance of antibody-specificity in determining successful treatment of radioimmunotherapy of B-cell Lymphoma”, (1999);94:(1) pp. 233-243.

14. Witzig T E, et al., "Treatment with ibritumomab tiuxetan radioimmunotherapy in patients with rituximab-refractory follicular non-Hodgkin's lymphoma”, J Clin Oncol (2002);20 (15): pp. 3,262-3,269.

15. Gordon, et al., "Treatment with ibritumomab tiuxetan ... long-term follow-up of a Phase I/II study", Blood (2004); 103 (12): $p p .4,429-4,431$.

16. Witzig, et al., "Safety of yttrium-90 ibritumomab tiuxetan radioimmunotherapy for relapsed low-grade, follicular, or transformed non-Hodgkin's lymphoma”, J Clin Oncol (2003);21:(7): pp. 1,263-1,270

17. Shipley, et al., Proc ASCO (2004); 23: 560, Abstract 6,519. 
as those with a reduced platelet count $(<150 \mathrm{x}$ $109 / \mathrm{L})$, need to be given a reduced dose of radioactivity. With this in mind, Zevalin $0.3 \mathrm{mCi} / \mathrm{kg}(11.1 \mathrm{MBq} / \mathrm{kg})$ proved to be both safe and efficacious. ${ }^{13-16}$

Although the results for single agent RIT are encouraging, the future is likely to involve integrating RIT into chemotherapy treatment protocols, and the current challenge for clinical investigators is to determine the optimal approach of integrating Zevalin RIT into chemotherapy schedules. Early data using Zevalin given to consolidate clinical responses following both shortened or full course chemotherapy look extremely promising. relapsed DLBC unsuitable for transplantation. ${ }^{18}$ Given this high single agent activity, clinical trials are now under way to integrate Zevalin into the front line treatment of DLBC and a large intergroup study with a randomisation for ${ }^{90} \mathrm{Y}$ ibritumomab tiuxetan (Zevalin) after full course R-CHOP chemotherapy is under way.

The dose-limiting toxicity from RIT is myelosuppression with delayed thrombocytopenia and neutropenia occurring at around four to six weeks after therapy. The extent and duration of the myelosuppression appears to depend on bone marrow reserve (amount of previous chemotherapy, age of patient), degree of bone marrow infiltration, pharmacokinetics of the $\mathrm{mAb}$,

\section{The dose-limiting toxicity from RIT is myelosuppression} with delayed thrombocytopenia and neutropenia occurring at around four to six weeks after therapy.

Shipley et al. presented impressive data demonstrating the ability of Zevalin to convert partial response after brief chemotherapy to complete responses. In this study patients were treated with four weekly infusions of Rituximab followed by three cycles of R-CHOP followed eight weeks later by Zevalin. Of the 22 patients who had completed the protocol there was a $40 \%$ CR rate before Zevalin, which was converted to a $86 \%$ CR rate after Zevalin. ${ }^{17}$

The large European intergroup study in previously untreated follicular lymphoma, which randomized patients to Zevalin after initial chemotherapy, has now completed accrual with over 400 patients recruited, and will provide invaluable data as to whether Zevalin may has a role as consolidation after primary chemotherapy.

Clinical responses have also been observed for Zevalin in transformed follicular and relapsed de novo diffuse large B-cell lymphoma (DLBC). Small numbers of patients were treated as part of the initial phase I/II study and response rates of $58 \%$ with a $33 \%$ CR rate were found. A recent European phase II trial with over 100 patients older than 60 with relapsed DLBC has confirmed this impressive response rate in large numbers of patients with and the stability of the radioimmunoconjugate.

Higher myeloablative doses may, however, be delivered safely with support from an autologous stem cell transplant. Zevalin RIT is currently being extensively investigated as a component of high dose therapy and Zevalin have been added to high dose chemotherapy with the 'standard' conditioning regimen of BCNU, Etoposide, Ara-C and Melphalan (BEAM). The early results confirm the feasibility of this approach and these important studies are on-going.

\section{Conclusion}

In summary, Zevalin RIT offers an excellent treatment alternative in relapsed follicular lymphoma and provides an attractive alternative in second relapse after R-Chemo schedules for many patients. Promising results are emerging in the treatment of aggressive lymphoma and RIT is now being integrated into treatment schedules for DLBC including high dose therapy and ASCT. The future for RIT is therefore likely to involve integration into chemotherapy schedules, and the recent clinical results suggests that this type of approach offers great promise for the future treatment of lymphoma. 